\title{
A Comparative Study of the Typhidot (Dot-EIA) versus Widal Test in Diagnosis of Typhoid Fever among Egyptian Patients
}

\author{
Rasha I. Salama1*, Nora M. Said ${ }^{2}$ \\ ${ }^{1}$ Tropical Medicine Department, Faculty of Medicine, Zagazig University, Zagazig, Egypt \\ ${ }^{2}$ Clinical Pathology Department, Faculty of Medicine, Zagazig University, Zagazig, Egypt \\ Email: *rashasalama1010@gmail.com,dr.nora2014@yahoo.com
}

How to cite this paper: Salama, R.I. and Said, N.M. (2019) A Comparative Study of the Typhidot (Dot-EIA) versus Widal Test in Diagnosis of Typhoid Fever among Egyptian Patients. Open Journal of Gastroenterology, 9, 91-98.

https://doi.org/10.4236/ojgas.2019.96011

Received: May 19, 2019

Accepted: June 24, 2019

Published: June 27, 2019

Copyright $\odot 2019$ by author(s) and Scientific Research Publishing Inc. This work is licensed under the Creative Commons Attribution International License (CC BY 4.0).

http://creativecommons.org/licenses/by/4.0/

\begin{abstract}
Background and Study Aim: Typhoid (Enteric) fever is a systemic infection caused by Salmonella Typhi and Salmonella Paratyphi. It is endemic in the developing countries including Egypt. Different diagnostic tools can achieve diagnosis and include cultures from the blood, stool, bone marrow, rarely urine for isolation of the organism. Antibody detection by Widal test and relatively recent typhoid are also used. The current study aimed at comparing the most commonly used antibody detection Widal test with the rapid antibody detection typhidot for diagnosis of typhoid fever among Egyptian adults. Patients and Methods: The study included 140 patients who are presented with picture suggestive of typhoid fever. Confirmed cases after the blood culture were included in the final analysis. Widal and typhidot tests were performed in all patients and were compared for sensitivity, specificity, positive predictive value, negative predictive value and diagnostic accuracy. Results: 45 patients out of 140 were diagnosed as typhoid fever by blood culture. Out of them, Widal test was positive in 39 patients with sensitivity, specificity, positive predictive value, negative predictive value and diagnostic accuracy of $86.7 \%, 89.5 \%, 79.5 \%, 93.4 \%$ and $88.5 \%$ respectively. Typhidot test was positive in 42 patients with sensitivity, specificity, positive predictive value, negative predictive value and diagnostic accuracy of 93.3\%, 90.6\%, 82.3\%, $96.6 \%$, and $91.4 \%$ respectively $(\mathrm{P}=0.00)$. Conclusions: Typhidot test is reliable, simple highly sensitive and specific test in diagnosing typhoid fever when compared with Widal test.
\end{abstract}

\section{Keywords}

Typhidot, Widal Test, Typhoid Fever 


\section{Introduction}

Typhoid (Enteric) fever, a systemic infection, is caused mainly by Salmonella typhi (S. typhi) and Salmonella paratyphi (S. paratyphi) [1]. It is a major public health problem and sometimes a life-threatening hazard to individuals in the developing world [2]. Although occur in all communities, it is endemic in the developing countries including Egypt. It's mainly transmitted by contaminated food and drinks [3] [4].

Diagnosis of typhoid fever is challenging because the disease had different stages while the infection is in evolution. Isolation of the organism from the blood is the routine method of diagnosis and less commonly from the stool and urine although sometimes challenging due to many causes including early introduction of antibiotics in the course of the disease and difficulty to isolate the organism from the blood in the first week of infection. Consequently, antibody detection by Widal test was routinely used a wide scale, because it is relatively cheaper, easy to perform and requires minimal training and equipment [5].

The classic Widal agglutination test detects the presence of antibodies against the somatic (O) and flagellar (H) antigens of Salmonella in the serum of infected patients. For accurate diagnosis of active typhoid infection, a four-fold rise in the titer (from 1:40 to 1:160) is needed [6]. However, such a rise is not always demonstrable. Also, it lacks sensitivity and specificity besides other limitations [7].

Typhidot has been proposed as a rapid and accurate serological test for the diagnosis of typhoid fever in comparison to Widal test [8]. But, a head to head comparison between both was not widely studied in the Egyptian literature. Consequently, this study aimed at comparing the most commonly used antibody detection Widal test with the rapid antibody detection typhidot for diagnosis of typhoid fever among Egyptian adults.

\section{Patients and Methods}

The study included 140 patients with acute febrile illness suggestive of typhoid fever infection presented to the outpatient clinic of the Tropical Medicine Department, Zagazig University Hospitals, Sharkia governorate, Egypt during the period from April 2018 to January 2019. They were 90 males and 50 females and their ages ranged from 15 to 50 years (mean age $32.25 \pm 10.5$ years). According to the blood culture results, they were divided into 2 groups. Group I included 45 patients with clinical diagnosis of typhoid fever confirmed by positive blood culture and group II comprised of 95 patients of suspected typhoid fever with negative blood culture for typhoidal salmonella.

\subsection{Inclusion Criteria}

All patients were with acute febrile illness of any age and sex suggestive of typhoid fever.

\subsection{Exclusion Criteria}

Patients who received antibiotic treatment for their symptoms or for any other 
reason, 2 weeks prior to the development of their current febrile illness besides patients unwilling to participate in the study were excluded from the study

Patient assessment: all patients were examined through history taking, full clinical examination and a battery of investigations, which comprised complete blood counts, urine analysis and culture, X-ray chest, liver function tests, blood culture, Widal test and tyhidot test. Results of blood culture, Widal, and typhidot test were compared in all patients for their sensitivity and specificity.

\subsection{Principle of Widal Test}

- The antibody in the serum produced in the response to Salmonella organism, the kit contains antigen suspension that is killed bacteria and they were stained to enhance the reading of agglutination tests.

- The blue stained antigens are specific to the somatic antigens (O-Ag), while the red stained antigens are specific to the flagella antigens $(\mathrm{H}-\mathrm{Ag})$.

\subsection{Procedures}

One drop each of undiluted patients' serum samples for the four antigens are placed on the circled card and one drop of each of the four Salmonella antigens are added separately and gently rotated for one minute. The appearance of agglutination gives qualitative results. To know the titer for each of the antigens, the test is repeated with dilutions of serum.

Typhidot test detects IgM and IgG antibodies against a specific $50 \mathrm{KD}$ antigen in the outer membrane protein (OMP) of the Salmonella typhi through a dot ELISA kit impregnated on nitrocellulose strips. The test becomes positive within 2 - 3 days of infection and separately identifies IgM and IgG antibodies. The reaction tray is divided into 2 columns marked as G and M. $250 \mathrm{ul}$ of sample diluent was dispensed in each well and $2.5 \mathrm{ul}$ of test/control was added and then incubated for 20 minutes. The strips were washed with washing buffer thrice, $250 \mathrm{ul}$ of anti-human IgG and IgM was dispensed then in each well and incubated for another 15 minutes. These were washed again, dispensed with $250 \mathrm{ul}$ of color development solution, and incubated for another 15 minutes and results were then interpreted. A positive IgM was interpreted clinically as acute typhoid illness, while IgM and IgG positive were taken as acute typhoid illness in the middle stage of infection and IgG positive was interpreted as a chronic carrier or previous infection or reinfection [9].

\section{Result}

In the current study, patients' age ranged between 15 and 50 years with a mean age $32.25 \pm 10.5$ years. Males were predominating (90/64.2\%) than females $(50 / 35.8 \%)$ as shown in (Table 1$)$. There was no statistically significant difference between all patients included in this study as regard age and gender $(\mathrm{P}=$ 0.154 and 0.12 respectively).

Typhoid fever was confirmed in 45 (32.1\%) out of the 140 (Group I) patients 
by their positive blood culture, whereas the remaining 95 patients (67.9\%) (Group II) were not positive in blood culture for typhoidal salmonella (Figure 1). A total of $49(35.0 \%)$ out of 140 patients tested positive for Widal test and 51 (36.5\%) out of 140 were positive for typhidot test.

There was a highly statistically significant association and agreement between Widal and typhidot tests among both studied groups. For Widal test (O-antigen) $(\mathrm{P}=0.00)$ and typhidot test $(\mathrm{P}=0.00)$ kappa agreement was 0.812 and 0.878 respectively (Table 2).

Table 1. Demographics of the studied patients.

\begin{tabular}{rrrr}
\hline & \multicolumn{2}{c}{ Age } \\
\hline Mean \pm SD & \multicolumn{2}{c}{$32.25 \pm 10.5$} \\
\cline { 2 - 3 } Median (Range) & \multicolumn{2}{c}{$33.0(15-50)$} \\
\cline { 2 - 4 } Sex & Nemale & 50 & 35.8 \\
& Male & 90 & 64.2 \\
Total & 140 & 100.0 \\
\hline
\end{tabular}

\section{Blood culture}

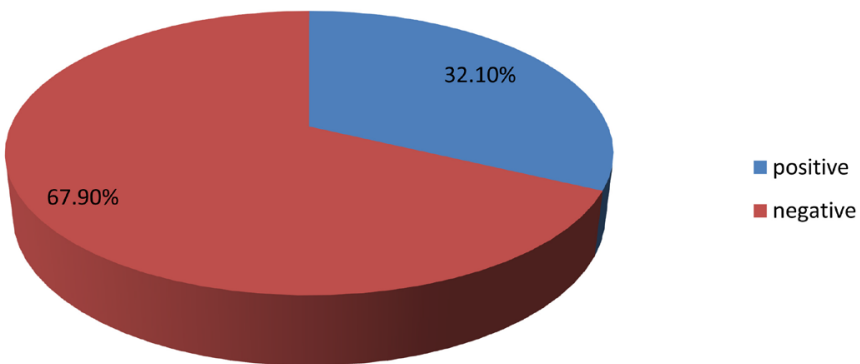

Figure 1. Result of the blood culture in the studied patients.

Table 2. Association and agreement of Widal and Typhidot tests.

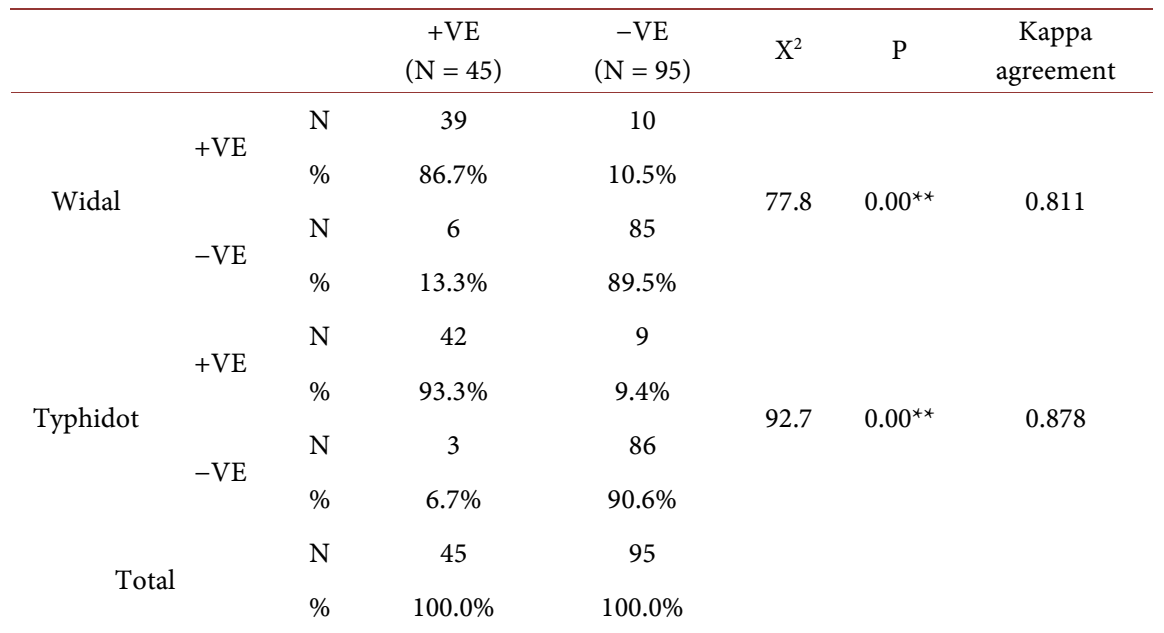

“**» Significant association and agreement. 
Among 45 patients in typhoid group, who were having positive blood culture, Widal test was positive in 39 patients, giving a sensitivity of $86.7 \%$, a specificity of $89.5 \%$, and a positive predictive value of $79.5 \%$ while Typhidot test was positive in 42 patients with sensitivity, specificity and positive predictive value of $93.3 \%, 90.6 \%$, and $82.3 \%$ respectively. Both tests showed a high statistically significant agreement with blood culture. Diagnostic accuracy of Widal was $88.5 \%$ while diagnostic accuracy for typhidot was $91.4 \%$ (Table 3 ).

\section{Discussion}

Therefore, a fast, reliable, and easy to perform the serodiagnostic test with a higher sensitivity and specificity than Widal test is required for rapid diagnosis and management of typhoid cases, thereby enabling clinicians to initiate an early therapy reducing morbidity and its complications. Typhidot is a rapid serological test for the diagnosis of typhoid fever. However, its usefulness in terms of specificity and sensitivity as compared to Widal test.

Typhoid fever is commonly encountered in less developed communities due to low socioeconomic level, bad general hygiene, lack of medical facilities and poor sanitation. The indiscriminate use of antibiotics leads to the endemicity of typhoid fever in different geographic locations with development of multi-resistant strains of Salmonella typhi raising significantly the morbidity and mortality related to typhoid fever in these communities [10] [11]. Furthermore, typhoid fever is a systemic disease difficult to diagnosis as the presenting symptoms are diverse [12]. Consequently, early and reliable diagnostic testing is required.

In the clinical practice, blood culture has remained the gold standard test in the diagnosis of typhoid fever, but it is difficult to use in early diagnosis due to difficult isolation of organism in early phases of illness. And that is why antibody detection by Widal test had been used for many years in the developing countries for diagnosing typhoid fever although it has many limitations. First, it has low sensitivity, specificity and positive predictive value, which changes with the geographical areas. Second, the sharing of $\mathrm{O}$ and $\mathrm{H}$ antigens by other Salmonella serotypes and other members of Enterobacteriaceae makes the role of Widal test even more controversial in diagnosing typhoid fever [13].

Reports from different geographic regions showed that a commercially available typhidot is an inexpensive and reliable serodiagnostic test for diagnosis of typhoid fever with higher sensitivity and specificity [14] and that is why we compared typhidot with Widal test for early and accurate diagnosis of typhoid fever among Egyptian patients.

Table 3. Diagnostic Validity of Widal and typhidot tests.

\begin{tabular}{cccccc}
\hline & Sensitivity & Specificity & $\begin{array}{c}\text { Positive Predictive } \\
\text { Value }\end{array}$ & $\begin{array}{c}\text { Negative Predictive } \\
\text { value }\end{array}$ & Accuracy \\
\hline Widal & $86.7 \%$ & $89.5 \%$ & $79.5 \%$ & $93.4 \%$ & $88.5 \%$ \\
Typhidot & $93.3 \%$ & $90.6 \%$ & $82.3 \%$ & $96.6 \%$ & $91.4 \%$ \\
\hline
\end{tabular}


In this study, typhidot showed better figures of sensitivity, specificity, positive and negative predictive values and overall diagnostic accuracy than that of Widal test in the diagnosis of typhoid against a reference of positive blood cultures and this would help early diagnosis and also early institution of therapy. In fact, similar favorable results were reported also from India, Pakistan and Malaysia. One study from India reported typhidot having a sensitivity of $100 \%$ and a specificity of $80 \%$ and was recommended for its utility in conjunction with Widal test for the early diagnosis of typhoid fever [15]. In another study group of typhoid patients in Pakistan, typhidot test had a comparable sensitivity of $94 \%$ and specificity of $77 \%$, while Widal test had a sensitivity and specificity of $63 \%$ and $83 \%$ only [16]. The effectiveness of typhidot test in early diagnosis of typhoid fever patients was also studied in two different studies in Malaysia. Its sensitivity and specificity was reported as $90.3 \%$ and $91.9 \%$ respectively in the first study, and was significantly higher, while the second study, also showed a sensitivity and specificity of $98 \%$ and $76.6 \%$ respectively [17] [18]. Both the Malaysian studies showed it to be a better test in contrast to Widal test for rapid diagnosis as well as for its simplicity of ease in use.

Due to low socioeconomic levels of patients, they used to get empirical antibiotics in case of fever without visiting doctors to get the right diagnosis and medication. Some patients in this study may deny taking antibiotics that leads to negative results.

\section{Conclusion}

Typhidot test is a sensitive and specific test in diagnosing typhoid fever. It is a rapid, easy to perform, more reliable test for typhoid fever as compared to Widal. However, culture isolation of Salmonella remains essential, especially for antibiotic susceptibility testing and these serological tests should be used in conjunction with culture for the early diagnosis of enteric fever [14] [19].

\section{Ethical Considerations}

The study protocol was carried out in concordance with the Medical Association Code of Ethics (declaration of Helsinki). The data were collected after written informed consent from all participants was obtained.

\section{Conflicts of Interest}

The authors declare no conflicts of interest regarding the publication of this paper.

\section{References}

[1] Ivanoff, B. (1995) Typhoid Fever, Global Situation and WHO Recommendations. Southeast Asian Journal of Tropical Medicine and Public Health, 26, 1-6.

[2] Raffatellu, M., Wilson, P., Winter, S.E., Andreas, J. and Bäumler, A.J. (2008) Clinical Pathogenesis of Typhoid Fever. The Journal of Infection in Developing Countries, 2, 260-266. https://doi.org/10.3855/jidc.219 
[3] Luby, S.P., Faizan, M.K., Fisher-Hoch, S.P., Syed, A., Mintz, E.D., Bhutta, Z.A., et al. (1998) Risk Factors for Typhoid Fever in an Endemic Setting, Karachi, Pakistan. Epidemiology \& Infection, 120, 129-138. https://doi.org/10.1017/S0950268897008558

[4] Gasem, M.H., Dolmans, W.M., Keuter, M.M. and Djokomoeljanto, R.R. (2001) Poor Food Hygiene and Housing as Risk Factors for Typhoid fever in Semarang, Indonesia. Tropical Medicine International Health, 6, 484-490. https://doi.org/10.1046/j.1365-3156.2001.00734.x

[5] Ley, B., Mtove, G., Thriemer, K., Thriemer, K., Amos, B., Seidlein, L.V., et al. (2010) Evaluation of the Widal Tube Agglutination Test for the Diagnosis of Typhoid Fever among Children Admitted to a Rural Hospital in Tanzania and a Comparison with Previous Studies. BMC Infectious Diseases, 10, 180.

https://doi.org/10.1186/1471-2334-10-180

[6] House, D., Chinh, N.T., Diep, T.S., Parry, C.M., Wain, J., Dougan, G., et al. (2005) Use of Paired Serum Samples for Serodiagnosis of Typhoid Fever. Journal of Clinical Microbiology, 43, 4889-4890. https://doi.org/10.1128/JCM.43.9.4889-4890.2005

[7] Olopoenia, L.A. and King, A.L. (2005) Widal Agglutination Test-100 Years Later: Still Plagued by Controversy. Postgrad Mserodiagnosis of Typhoid Fever. Journal of Clinical Microbiology, 43, 4889-4890.

[8] Jyoti Kumar Dinkar, Naresh Kumar, Chandrakishore (2017) A Comparative Study of Widal Test and Typhidot (IgM and IgG Specific Assay) Test in the Diagnosis of Enteric Fever. International Association of Infant Massage, 4, 126-130.

[9] Sherwal, B.L., Dhamija, R.K., Randhawa, V.S., Jais, M., Kaintura, A. and Kumar, M. (2004) A Comparative Study of Typhidot and Widal Test in Patients of Typhoid Fever. Journal Indian Academy of Clinical Medicine, 5, 244-246.

[10] Brown, J.C., Shanahan, P.M., Jesudason, M.V., et al. (1996) Mutations Responsible for Reduced Susceptibility to 4-Quinilones in Clinical Isolates of Multi-Resistant Salmonella typhi in India. Journal of Antimicrobial Chemotherapy, 37, 891-900. https://doi.org/10.1093/jac/37.5.891

[11] Therlfall, E.J., Ward, L.R., Skinner, J.A., Smith, H.R. and Lacy, S. (1999) Ciprofloxacin Resistant Salmonella typhi and Treatment Failure. The Lancet, 353, 1590-1591. https://doi.org/10.1016/S0140-6736(99)01001-6

[12] Stuart, B.M. and Pullen, R.L. (1946) Typhoid Fever: Clinical Analysis of Three Hundred and Sixty Cases. Archives of Internal Medicine, 78, 629-661. https://doi.org/10.1001/archinte.1946.00220060002001

[13] Parry, C.M., Hien, T.T., Dougan, G., et al. (2002) Typhoid Fever. The New England Journal of Medicine, 347, 1770-1782. https://doi.org/10.1056/NEJMra020201

[14] Wijedoru, L., Mallett, S. and Parry, C.M. (2017) Rapid Diagnostic Tests for Typhoid and Paratyphoid (Enteric) Fever. Cochrane Database of Systematic Reviews, No. 5, CD008892. https://doi.org/10.1002/14651858.CD008892.pub2

[15] Jesudasson, M., Esther, E. and Mathai, E. (2002) Typhidot Test to Detect IgG and IgM Antibodies in Typhoid Fever. The Indian Journal of Medical Research, 116, 70-72.

[16] Butta, Z.A. and Mansurali, N. (1999) Rapid Serological Diagnosis of Pediatric Typhoid Fever in an Endemic Area: A Prospective Comparative Evaluation of Two Dot-Enzyme Immunoassays and the Widal Test. The American Journal of Tropical Medicine and Hygiene, 61, 645-647. https://doi.org/10.4269/ajtmh.1999.61.654

[17] Choo, K.E., Davis, T.M., Ismail, A., et al. (1999) Rapid and Reliable Serological Diagnosis of Enteric Fever: Comparative Sensitivity and Specificity of Typhidot and 
Typhidot-M in Febrile Malaysian Children. Acta Tropica, 72, 175-183. https://doi.org/10.1016/S0001-706X(98)00095-3

[18] Gopalakrishan, V., Sekhar, W.Y., Soo, E.H., et al. (2002) Typhoid Fever in Kuala Lumpur and a Comparative Evaluation of Two Commercially Available Diagnostic Kits for Detection of Antibodies to Salmonella typhi. Singapore Medical Journal, 43, 354-358.

[19] Ajibola, O., Mshelia, M.B., Gulumbe, B.H. and Eze, A.A. (2018) Typhoid Fever Diagnosis in Endemic Countries: A Clog in the Wheel of Progress. Medicina, 54, 23. https://doi.org/10.3390/medicina54020023 